\title{
THE MATHEMATICAL THEORY OF LOW MACH NUMBER FLOWS
}

\author{
Steven Schochet ${ }^{1}$
}

\begin{abstract}
The mathematical theory of the passage from compressible to incompressible fluid flow is reviewed.
\end{abstract}

Mathematics Subject Classification. 35Q30, 35Q35, 76G25.

Plenary lecture, Low Mach Number Flows Conference, June 21-25, 2004, Porquerolles, France.

\section{INTRODUCTION}

Incompressible fluid flow differs from compressible fluid flow in that one of the equations of evolution is replaced by the constraint that the flow be divergence-free. The mathematical theory of low Mach number flow attempts to bridge the gap between those two different descriptions of fluid flow by determining in what sense compressible flows tend to incompressible ones as the Mach number tends to zero. That theory will be reviewed here using as a starting point the physically inconsistent (cf. [34], pp. 14-15) but mathematically convenient equations

$$
\begin{aligned}
\rho_{t}+\nabla \cdot(\rho \mathcal{U}) & =0 \\
\rho \mathcal{U}_{t}+\rho \mathcal{U} \cdot \nabla \mathcal{U}+\nabla \mathcal{P} & =\mu \Delta \mathcal{U}+(\mu+\lambda) \nabla(\nabla \cdot \mathcal{U}) \\
\mathcal{S}_{t}+\mathcal{U} \cdot \mathcal{S} & =0
\end{aligned}
$$

for compressible flow. Here $\rho$ is the density of the fluid, $\mathcal{U}$ its velocity, $\mathcal{P}$ the pressure, and $\mathcal{S}$ the specific entropy. Equations (1.1)-(1.3) must be supplemented by an equation of state giving one of the thermodynamical variables $\rho, \mathcal{P}$, or $\mathcal{S}$ in terms of the other two. For simplicity the viscosity coefficients $\mu$ and $\mu+\lambda$ have been taken to be nonnegative constants. In essence, this framework allows us to treat simultaneously the three most commonlystudied models: the isentropic compressible Euler equations, for which $\mu=0=\lambda$ and $\mathcal{P}$ depends solely on $\rho$ so that the equation for $\mathcal{S}$ is not needed, the non-isentropic compressible Euler equations, for which the viscosity coefficients still vanish but the equation of state is generalized to $\rho=\rho(\mathcal{P}, \mathcal{S})$, and the barotropic compressible Navier-Stokes equations, for which the equation of state relating $\mathcal{P}$ to $\rho$ is retained and the entropy equation is again omitted but the viscosity coefficients $\mu$ and $\mu+\lambda$ are positive.

After rescaling equations (1.1)-(1.3) so as to introduce a global Mach number or compressibility parameter, taking the limit as that parameter tends to zero yields, at least formally, equations for incompressible flow. The exact form of those equations depends on certain additional assumptions about the scales of the problem

Keywords and phrases. Incompressible limit, Mach number.

1 School of Mathematical Sciences, Tel Aviv University. schochet@post.tau.ac.il

(C) EDP Sciences, SMAI 2005 
introduced by the rescaling procedure and the initial data. Various cases and extensions of the theory will be described below.

\section{EARLY HiSTORY OF THE THEORY OF LOW-MACH-NUMBER FLOW}

The first theory of low Mach number flow, due to Janzen and Rayleigh (see [41], Sect. 47, [51]) for expositions and references) dealt with steady irrotational flow. Their expansion in powers of the Mach number was used both as a computational tool and as a method for proving the existence of compressible flow. Sirovich [47] extended the use of such expansions to non-steady flows, albeit on the time scale of the fast acoustic waves rather than the slower scale of the flow of the fluid particles. The effect of slight compressibility on that longer scale was first considered in the context of the numerical method of artificial compressibility [9, 48], in which the true equation(s) for the evolution of the thermodynamical variables are replaced by a simpler linear model equation for $P$.

The first general proof of the convergence of compressible fluid flow to incompressible flow was given by Ebin [14] using a differential-geometric formulation that models constraints as a limit of large potentials in dynamical systems. This incompressible limit and other singular limits were formulated directly in terms of partial differential equations by Kreiss [7,32] using the bounded derivative method, which employs transformations to normal forms and places severe restrictions on the initial data, although those restrictions were later relaxed [6]. Finally, Klainerman \& Majda [28] proved the convergence of compressible to incompressible flow by directly obtaining estimates for the scaled form of the partial differential equations; their approach has been followed in most subsequent work.

\section{SCALING}

\subsection{Dimensional scaling}

The Mach number can be introduced into the equations in at least two different ways. First, we may consider fluids having varying equations of state $(e . g$. [14]) and, for viscous fluids, also varying viscosity coefficients. From this point of view one thinks of a particular fluid, such as water perhaps, as being "nearly incompressible". Alternatively or in addition, we can consider flows of varying sizes on varying time and spatial scales. One then thinks of certain flows as having a low global Mach number, although other flows of the same fluid may be quite compressible. We shall use the latter procedure here since it shows that any fluid may exhibit nearly incompressible behavior.

Furthermore, introducing the Mach number via scaling is a richer procedure that can accommodate more complex flows because it is possible to include more than one scale for any dimension. In particular, following $[30,37]$ we shall consider flows that may have two length scales $L_{1}$ and $L_{2}$, where

$$
\delta:=\frac{L_{1}}{L_{2}} \ll 1
$$

For the moment a single velocity scale $U$ will be used; adding additional velocity scales is essentially equivalent to making an asymptotic expansion in the dependent variables, which will be considered later. Although, anticipating that $U$ will be small, we could consider velocities of the form $\mathcal{U}=\mathcal{U}_{0}+U \mathbf{u}(t, \mathbf{x})$ rather than just

$$
\mathcal{U}=U \mathbf{u}(t, \mathbf{x})
$$

the added constant velocity $\mathcal{U}_{0}$ can be eliminated by using the Galilean invariance of system (1.1)-(1.3). For the pressure, however, a leading constant term cannot be eliminated, so we will take the pressure to have the form

$$
\mathcal{P}=\mathcal{P}_{0}+P p(t, \mathbf{x}) .
$$


Although we shall soon see that $P$ should be small, (3.3) still allows for the possibilities that $P=1$ with $\mathcal{P}_{0}>\max |p|$ or even that $P$ is large, in which case $\mathcal{P}_{0}$ may as well be taken to equal to zero. However, to the extent that we are still concerned with the physical accuracy of the system under discussion, scalings for which $\mathcal{P}$, and hence also $\rho$, takes small or large values cause difficulties because the viscosity coefficients then become pressure-dependent [50]. Taking $\mathcal{P}_{0}$ nonzero and $P$ sufficiently small so that the pressure remains bounded away from zero avoids this problem. Since the sound speed is then of order one, the Mach number $M$, defined as the maximum of the ratio of absolute fluid velocity to sound speed, then satisfies

$$
M=O(U)
$$

All the preceding scalings of the independent and dependent variables are essentially conditions on sequences of initial data. For example, for the case of a single length scale $L$ and velocity scale $U$, the initial velocity $\mathcal{U}_{0}(\mathcal{X})$ should be such that the non-dimensionalized velocity $u_{0}(\mathbf{x}):=\frac{\mathcal{U}_{0}(L \mathbf{x})}{U}$ is uniformly bounded in some appropriate space. In contrast, the appropriate time scale(s) are determined not just by the initial data but by the system of PDEs.

\subsection{Determination of the time scales}

Although substituting the scalings of all the variables into (1.1)-(1.3) would lead to rather long equations, we only need to know the sizes of the resulting terms in order to determine the appropriate time scales. Because the scaling of the density depends on the form assumed for the equation of state, it is convenient to consider instead the scaling of the pressure equation

$$
\frac{1}{\rho} \frac{\partial \rho}{\partial \mathcal{P}}\left[\mathcal{P}_{t}+\mathcal{U} \cdot \nabla \mathcal{P}\right]+\nabla \cdot \mathcal{U}=0
$$

derived from (1.1) and (1.3). Although it is usually most convenient to non-dimensionalize equations when scaling them, since we are trying to discover the appropriate time scales it is more convenient here to rescale the equations to all have the same dimension time ${ }^{-1}$. For the momentum equation (1.2) this can be accomplished by multiplying by $\frac{1}{\bar{\rho} U}$, where $\bar{\rho}=O(1)$ is a typical density and $U$ is given by (3.2). Although the pressure (3.5) has already been formulated so as to have that dimensionality, it will nevertheless be convenient to multiply it by the non-dimensional factor $\frac{\mathcal{P}_{0}}{P}$, where $\mathcal{P}_{0}$ and $P$ are given in (3.3). The non-dimensional factor $\frac{\mathcal{P}_{0}}{\rho} \frac{\partial \rho}{\partial \mathcal{P}}$ appearing in the resulting pressure equation is of order one, and the dimensional factors $\mathcal{P}_{0}, 1 / \bar{\rho}$, and $\nu:=\mu / \bar{\rho}$ appearing in the resulting pressure or momentum equations have independent dimensions, whose units may therefore be chosen to make them all also of order one. In the following, any equation between quantities of different dimensions is understood to hold after multiplying one side by an appropriate combination of those $O(1)$ dimensional quantities. The terms appearing in the rescaled equations then have the following sizes:

$$
\begin{array}{cccccc}
\text { Type of Term: } & \text { Time Derivative } & \text { Convection } & \text { Pressure } & \text { Compression } & \text { Dissipation } \\
\text { Original Form: } & \frac{1}{\rho} \frac{\partial \rho}{\partial \mathcal{P}} \mathcal{P}_{t}, \rho \mathcal{U}_{t} & \frac{1}{\rho} \frac{\partial \rho}{\partial \mathcal{P}} \mathcal{U} \cdot \nabla \mathcal{P}, \rho \mathcal{U} \cdot \nabla \mathcal{U} & \nabla \mathcal{P} & \nabla \cdot \mathcal{U} & \mu \Delta \mathcal{U},(\mu+\lambda) \nabla(\nabla \cdot \mathcal{U}) \\
\text { Scaled Sizes: } & O\left(\frac{1}{T_{j}}\right) & O\left(\frac{U}{L_{j}}\right) & O\left(\frac{P}{U L_{j}}\right) & O\left(\frac{U}{P L_{j}}\right) & O\left(\frac{1}{L_{j} L_{k}}\right)
\end{array}
$$

Since every rescaled term has the dimension time ${ }^{-1}$, each of the last four sizes in the table determines a time scale, at least after normalizing by the $O(1)$ dimensional constants as discussed above. In particular, let us define $T_{1}$ to be the convective time

$T_{2}$ to be the pressure, or acoustic time

$$
T_{1}:=\frac{L_{1}}{U}
$$

$$
T_{2}:=\frac{U L_{1}}{P},
$$


and $\varepsilon$ to be the ratio of those time scales, i.e.,

$$
\varepsilon:=\frac{T_{2}}{T_{1}}=\frac{U^{2}}{P}
$$

After non-dimensionalizing the pressure and momentum equations by multiplying by $T_{1}$, the terms in those equations have the sizes:

$$
\begin{array}{cccccc}
\text { Type of Term: } & \text { Time Derivative } & \text { Convection } & \text { Pressure } & \text { Compression } & \text { Dissipation } \\
\text { Normalized Sizes: } & 1, \frac{1}{\varepsilon} & 1, \delta & \frac{1}{\varepsilon}, \frac{\delta}{\varepsilon} & \frac{1}{P}, \frac{\delta}{P} & \frac{1}{U L_{1}}, \frac{\delta}{U L_{1}}, \frac{\delta^{2}}{U L_{1}}
\end{array}
$$

where $\delta$ and $\varepsilon$ are defined in (3.1) and (3.6), respectively.

\subsection{Rich scaling}

As noted above, as long as $\mathcal{P}_{0}=O(1)$ and $P$ is at most $O(1)$, the Mach number is essentially the velocity scale $U$, and these together imply that for low Mach number flow $\varepsilon \ll 1$. The non-dimensionalized equations therefore formally yield separate equations on at least the two scales $O\left(\frac{1}{\varepsilon}\right)$ and $O(1)$. As for the terms whose size in relation to powers of $\varepsilon$ has not yet been fixed, any term that is $\gg O(1)$ but not exactly $O\left(\frac{1}{\varepsilon}\right)$ would produce a separate equation requiring a new time scale, while any term that is $\ll O(1)$ is negligible to leading order.

The most interesting choices of the remaining parameters are the "rich limits", which have as many terms as possible having sizes $O\left(\frac{1}{\varepsilon}\right)$ or $O(1)$, since those terms are then included in the equations on those scales. In particular, in order to make the term $O\left(\frac{\delta}{\varepsilon}\right)$ be of order one, we choose the length ratio to be

$$
\delta=\varepsilon .
$$

The richest choice of the pressure variation scale is then

$$
P=\varepsilon,
$$

which makes each of the pressure scales equal one of the scales $O\left(\frac{1}{\varepsilon}\right)$ or $O(1)$ already present. Moreover, the alternative scaling $P=O(1)$, which would make one pressure scale equal a scale already present, seems to make the initial value problem not be uniformly well posed, because the symmetrizer of the resulting equations is $O\left(\frac{1}{\varepsilon}\right)$ rather than $O(1)$.

Since (3.6) plus (3.8) imply that

$$
U=\varepsilon,
$$

(3.4) then shows that $\varepsilon$ equals the Mach number up to an $O(1)$ factor.

Turn next to the choice of the length scale: Although the choices $L_{1}=1$ or $L_{1}=\varepsilon$ are the richest since in view of (3.7) and (3.9) they makes viscous terms appear on both the $O\left(\frac{1}{\varepsilon}\right)$ and $O(1)$ scales, the presence of viscosity on the fast $O\left(\frac{1}{\varepsilon}\right)$ time scale would make the solution dissipate to zero before the convective $O(1)$ time scale was reached. We therefore take

$$
L_{1}=\frac{1}{\varepsilon}
$$

if viscosity is present. In the inviscid case the basic length scale $L_{1}$ remains arbitrary.

\subsection{Multiple-scale scaled equations}

Assuming for convenience that (3.10) holds even in the inviscid case, the above rescalings of the dependent and independent variables all have the form

$$
\mathcal{V}(\mathcal{T}, \mathcal{X})=\mathcal{V}_{0}+\varepsilon \tilde{v}\left(\varepsilon^{2} \mathcal{T}, \varepsilon \mathcal{X}\right), \quad \tilde{v}(t, x)=v\left(t, \frac{t}{\varepsilon}, \mathbf{x}, \varepsilon \mathbf{x}\right)=\left.v(t, \tau, \mathbf{x}, \mathbf{y})\right|_{\tau=\frac{t}{\varepsilon}, \mathbf{y}=\varepsilon \mathbf{x}} .
$$


In order to obtain the scaled equations we will not only substitute rescalings of the form (3.11) into the original PDEs, but we will also assume that the resulting equations hold for all values of $\mathbf{y}$ and all nonnegative values of $\tau$, not only when those variables equal $\varepsilon \mathbf{x}$ and $t / \varepsilon$, respectively. As a consequence, the rescaled PDEs so obtained involve multiple time and spatial variables. Although the validity of those rescaled equations is therefore not necessary in order for the original equations to hold, it is certainly sufficient.

Since the set of dependent variables used in the barotropic and non-isentropic cases differ, we consider the resulting equations separately for those two cases.

\subsubsection{Barotropic viscous flow}

When the equation of state has the form $\mathcal{P}=\mathcal{P}(\rho)$, then (3.3) and (3.8) imply that the density has the form

$$
\rho=\rho_{0}+\varepsilon r(t, x)
$$

and it is then convenient to take $\bar{\rho}$ to equal $\rho_{0}$. Substituting (3.9) into (3.2), extending the result and (3.12) to multiple independent variables of the form (3.11), and substituting the results into (1.1)-(1.2) yields

$$
\frac{1}{\varepsilon} r_{\tau}+r_{t}+\mathbf{u} \cdot \nabla_{\mathbf{x}} r+\varepsilon \mathbf{u} \cdot \nabla_{\mathbf{y}} r+\frac{\left(\rho_{0}+\varepsilon r\right)}{\varepsilon} \nabla_{\mathbf{x}} \cdot \mathbf{u}+\left(\rho_{0}+\varepsilon r\right) \nabla_{\mathbf{y}} \cdot \mathbf{u}=0
$$

and

$$
\begin{aligned}
& \left(\rho_{0}+\varepsilon r\right)\left[\frac{1}{\varepsilon} \mathbf{u}_{\tau}+\mathbf{u}_{t}+\mathbf{u} \cdot \nabla_{\mathbf{x}} \mathbf{u}+\varepsilon \mathbf{u} \cdot \nabla_{\mathbf{y}} \mathbf{u}\right]+\frac{\mathcal{P}^{\prime}\left(\rho_{0}+\varepsilon r\right)}{\varepsilon} \nabla_{\mathbf{x}} r+\mathcal{P}^{\prime}\left(\rho_{0}+\varepsilon r\right) \nabla_{\mathbf{y}} r \\
& \quad=\mu\left[\Delta_{\mathbf{x}} \mathbf{u}+2 \varepsilon \nabla_{\mathbf{x}} \cdot \nabla_{\mathbf{y}} \mathbf{u}+\varepsilon^{2} \Delta_{\mathbf{y}} \mathbf{u}\right]+(\mu+\lambda)\left[\nabla_{\mathbf{x}} \nabla_{\mathbf{x}} \cdot \mathbf{u}+\varepsilon\left(\nabla_{\mathbf{x}} \nabla_{\mathbf{y}} \cdot \mathbf{u}+\nabla_{\mathbf{y}} \nabla_{\mathbf{x}} \cdot \mathbf{u}\right)+\varepsilon^{2} \nabla_{\mathbf{y}} \nabla_{\mathbf{y}} \cdot \mathbf{u}\right] .
\end{aligned}
$$

\subsubsection{Non-isentropic inviscid flow}

Since the equation for the entropy is linear in $\mathcal{S}$, the scale $S$ of the entropy variation is arbitrary, and will be assumed here to be $S=1$ so that the transformation for the entropy corresponding to (3.3) or (3.2) is simply $\mathcal{S}=s$. Substituting (3.8) and (3.9) into those relations, extending as in (3.11), and substituting the results and the equation of state $\rho=\rho(\mathcal{P}, \mathcal{S})$ into $(3.5)$ and $(1.2)-(1.3)$ yields

$$
\begin{array}{r}
\frac{1}{\rho} \frac{\partial \rho}{\partial \mathcal{P}}\left[\frac{1}{\varepsilon} \partial_{\tau} p+\partial_{t} p+\mathbf{u} \cdot \nabla_{\mathbf{x}} p+\varepsilon \mathbf{u} \cdot \nabla_{\mathbf{y}} p\right]+\frac{1}{\varepsilon} \nabla_{\mathbf{x}} \cdot \mathbf{u}+\nabla_{\mathbf{y}} \cdot \mathbf{u}=0 \\
\rho\left[\frac{1}{\varepsilon} \partial_{\tau} \mathbf{u}+\partial_{t} \mathbf{u}+\mathbf{u} \cdot \nabla_{\mathbf{x}} \mathbf{u}+\varepsilon \mathbf{u} \cdot \nabla_{\mathbf{y}} \mathbf{u}\right]+\frac{1}{\varepsilon} \nabla_{\mathbf{x}} p+\nabla_{\mathbf{y}} p=0 \\
\frac{1}{\varepsilon} \partial_{\tau} s+\partial_{t} s+\mathbf{u} \cdot \nabla_{\mathbf{x}} s+\varepsilon \mathbf{u} \cdot \nabla_{\mathbf{y}} s=0
\end{array}
$$

where $\rho$ and its derivative are evaluated at $\left(\mathcal{P}_{0}+\varepsilon p, s\right)$.

\section{Ansatz And formal Limit}

\subsection{Multiple-scale expansion formalism}

The simplest way to derive the equations satisfied in the limit when $\varepsilon \rightarrow 0$ is to first expand all the dependent variables in powers of $\varepsilon$ as functions of the multiple independent variables ( $c f .[4,29,37,40,52])$ :

$$
v=v^{0}(t, \tau, y, x)+\varepsilon v^{1}(t, \tau, y, x)+\ldots,
$$

and then expand the resulting equations in powers of $\varepsilon$ as well. Even though the expansion (4.1) may not always be valid (due to small-divisor or other problems), it is, as noted in [26], still useful for deriving the correct limit equations. In particular, it will not be sufficient to consider only the zeroth-order term $v^{0}$. 
To simplify the presentation, the barotropic, possibly viscous, equations (3.13)-(3.14) will be considered first. Substituting expansions of the form (4.1) for $r$ and $\mathbf{u}$ into those equations yields

$$
\begin{aligned}
r_{\tau}^{0}+\rho_{0} \nabla_{\mathbf{x}} \cdot \mathbf{u}^{0} & =0 \\
\rho_{0} \mathbf{u}_{\tau}^{0}+\mathcal{P}^{\prime}\left(\rho_{0}\right) \nabla_{\mathbf{x}} r^{0} & =0
\end{aligned}
$$

as the $O\left(\frac{1}{\varepsilon}\right)$ equations, and

$$
\begin{gathered}
r_{\tau}^{1}+\rho_{0} \nabla_{\mathbf{x}} \cdot \mathbf{u}^{1}+r_{t}^{0}+\mathbf{u}^{0} \cdot \nabla_{\mathbf{x}} r^{0}+r^{0} \nabla_{\mathbf{x}} \cdot \mathbf{u}^{0}+\rho_{0} \nabla_{\mathbf{y}} \cdot \mathbf{u}^{0}=0 \\
\rho_{0} \mathbf{u}_{\tau}^{1}+\mathcal{P}^{\prime}\left(\rho_{0}\right) \nabla_{\mathbf{x}} r^{1}+r^{0} u_{\tau}^{0}+\rho_{0}\left[\mathbf{u}_{t}^{0}+\mathbf{u}^{0} \cdot \nabla_{\mathbf{x}} \mathbf{u}^{0}\right]+\mathcal{P}^{\prime \prime}\left(\rho_{0}\right) r^{0} \nabla_{\mathbf{x}} r^{0}+\mathcal{P}^{\prime}\left(\rho_{0}\right) \nabla_{\mathbf{y}} r^{0} \\
=\mu \Delta_{\mathbf{x}} \mathbf{u}^{0}+(\mu+\lambda) \nabla_{\mathbf{x}} \nabla_{\mathbf{x}} \cdot \mathbf{u}^{0}
\end{gathered}
$$

as the $O(1)$ equations.

\subsection{Sublinear growth condition}

As is typical of singular perturbation problems, the $O(1)$ equations involve the first-order perturbations $r^{1}$ and $\mathbf{u}^{1}$, which therefore need to be eliminated in order to obtain a closed set of equations for the limit solution $r^{0}$ and $\mathbf{u}^{0}$. The standard method to do so is by the sublinear growth condition, i.e., the condition that the first-order perturbation terms be $o(\tau)$, so that the ordering of the expansion remain correct up through the value $O\left(\frac{1}{\varepsilon}\right)$ of the fast time $\tau$ actually occurring in the expansion (3.11). Even when the zeroth-order solution is independent of $\tau$, which is known as the slow case, the first-order perturbations generally will depend on that variable, so that the sublinear growth condition remains relevant.

In order to calculate the sublinear growth condition, define the full vector of solution components

$$
\mathbf{v}:=\left(\begin{array}{l}
r \\
\mathbf{u}
\end{array}\right)
$$

the "fast" operator

$$
\mathbb{L}:=-\left(\begin{array}{cc}
0 & \rho_{0} \nabla_{\mathbf{x}} \\
\frac{\mathcal{P}^{\prime}\left(\rho_{0}\right)}{\rho_{0}} \nabla_{\mathbf{x}} & 0
\end{array}\right)
$$

that allows the $O\left(\frac{1}{\varepsilon}\right)$ equations (4.2)-(4.3) to be written succinctly as

$$
\mathbf{v}_{\tau}^{0}=\mathbb{L} \mathbf{v}^{0}
$$

the solution operator

$$
\mathbb{S}(\tau):=\mathrm{e}^{\tau \mathbb{L}}=\mathbb{F}_{x}^{-1} \mathrm{e}^{\tau\left(\begin{array}{cc}
0 & -i \rho_{0} \mathbf{k} \cdot \\
-i \frac{\mathcal{P}^{\prime}\left(\rho_{0}\right)}{\rho_{0}} \mathbf{k} & 0
\end{array}\right)} \mathbb{F}_{x}
$$

of (4.7), where $\mathbb{F}_{x}$ denotes the Fourier transform with respect to $\mathbf{x}$ with dual variable $\mathbf{k}$, and the averaging operator

Then the $O(1)$ equations (4.4)-(4.5) have the form

$$
\mathbb{M}_{\tau}[f]:=\lim _{\tau \rightarrow \infty} \frac{1}{\tau} \int_{0}^{\tau} f\left(t, \tau^{\prime}, x, y\right) \mathrm{d} \tau^{\prime} .
$$

$$
\mathbf{v}_{\tau}^{1}-\mathbb{L} \mathbf{v}^{1}=\mathbf{f}^{0},
$$

where

$$
\mathbf{f}^{0}:=-\left(\begin{array}{c}
r_{t}^{0}+\mathbf{u}^{0} \cdot \nabla_{\mathbf{x}} r^{0}+r^{0} \nabla_{\mathbf{x}} \cdot \mathbf{u}^{0}+\rho_{0} \nabla_{\mathbf{y}} \cdot \mathbf{u}^{0} \\
\frac{r^{0}}{\rho_{0}} u_{\tau}^{0}+\mathbf{u}_{t}^{0}+\mathbf{u}^{0} \cdot \nabla_{\mathbf{x}} \mathbf{u}^{0}+\frac{\mathcal{P}^{\prime \prime}\left(\rho_{0}\right)}{\rho_{0}} r^{0} \nabla_{\mathbf{x}} r^{0}+\frac{\mathcal{P}^{\prime}\left(\rho_{0}\right)}{\rho_{0}} \nabla_{\mathbf{y}} r^{0}-\frac{\mu}{\rho_{0}} \Delta_{\mathbf{x}} \mathbf{u}^{0}-\frac{\mu+\lambda}{\rho_{0}} \nabla_{\mathbf{x}} \nabla_{\mathbf{x}} \cdot \mathbf{u}^{0}
\end{array}\right) .
$$


The solution $\mathbf{v}^{1}$ to $(4.9)$ can be written as

$$
\begin{aligned}
v^{1} & =\mathbb{S}(\tau) v^{1}(t, 0, \mathbf{x}, \mathbf{y})+\int_{0}^{\tau} \mathbb{S}\left(\tau-\tau^{\prime}\right) f\left(t, \tau^{\prime}, \mathbf{x}, \mathbf{y}\right) \mathrm{d} \tau^{\prime} \\
& =\mathbb{S}(\tau)\left[v^{1}(t, 0, \mathbf{x}, \mathbf{y})+\int_{0}^{\tau} \mathbb{S}\left(-\tau^{\prime}\right) f\left(t, \tau^{\prime}, \mathbf{x}, \mathbf{y}\right) \mathrm{d} \tau^{\prime}\right] .
\end{aligned}
$$

Since $\tilde{A} \mathbb{L}$ is antisymmetric, where

$$
\tilde{A}=\left(\begin{array}{cc}
\frac{1}{\rho_{0}} & \mathbf{0} \\
\mathbf{0} & \frac{\rho_{0}}{\mathcal{P}^{\prime}\left(\rho_{0}\right)} I
\end{array}\right),
$$

the solution operator $\mathbb{S}$ is unitary with respect to the inner product

$$
(\mathrm{g}, \tilde{A} \mathbf{h}):=\int \mathbf{g}^{T} \tilde{A} \mathbf{h} \mathrm{d} \mathbf{x}
$$

which implies that $\mathbf{v}^{1}$ is sublinear if $\mathbb{S}(-\tau) \mathbf{v}^{1}$ is. In addition, $\mathbf{v}(t, 0, x, y)$ does not depend on $\tau$, so the sublinearity condition $\lim _{\tau \rightarrow \infty} \frac{1}{\tau} \mathbf{v}^{1}=0$ reduces to the condition that

$$
0=\lim _{\tau \rightarrow \infty} \frac{1}{\tau} \int_{0}^{\tau} \mathbb{S}\left(-\tau^{\prime}\right) \mathbf{f}^{0}\left(t, \tau^{\prime}, \mathbf{x}, \mathbf{y}\right) \mathrm{d} \tau^{\prime}=\mathbb{M}_{\tau}\left[\mathbb{S}(-\tau) \mathbf{f}^{0}\right]
$$

Using once more the fact that $\mathbb{S}$ is unitary, condition (4.11) can be written in the form

$$
0=\mathbb{E}\left[\mathbf{f}^{0}\right]:=\mathbb{S}(\tau) \mathbb{M}_{\tau}\left[\mathbb{S}(-\tau) \mathbf{f}^{0}\right]
$$

which is convenient because the operator $\mathbb{E}$ is a projection. Specifically [44], $\mathbb{E}$ is the projection operator onto terms of the form

$$
\mathbb{S}(\tau) \mathbf{f}(t, \mathbf{x}, \mathbf{y})
$$

with respect to the inner product

$$
\langle\mathbf{g}, \mathbf{h}\rangle:=\lim _{\tau \rightarrow \infty} \frac{1}{\tau} \int_{0}^{\tau}(\mathbf{g}, \tilde{A} \mathbf{h}) \mathrm{d} \tau^{\prime} .
$$

Since the $O\left(\frac{1}{\varepsilon}\right)$ equations (4.7) say that $v^{0}$ has the form (4.13), this alternative characterization of $\mathbb{E}$ yields

$$
\mathbf{v}^{0}=\mathbb{E} \mathbf{v}^{0}
$$

while combining (4.12) with (4.10) yields

$$
\left(\begin{array}{l}
0 \\
0
\end{array}\right)=\mathbb{E}\left(\begin{array}{c}
r_{t}^{0}+\mathbf{u}^{0} \cdot \nabla_{\mathbf{x}} r^{0}+r^{0} \nabla_{\mathbf{x}} \cdot \mathbf{u}^{0}+\rho_{0} \nabla_{\mathbf{y}} \cdot \mathbf{u}^{0} \\
\frac{r^{0}}{\rho_{0}} u_{\tau}^{0}+\mathbf{u}_{t}^{0}+\mathbf{u}^{0} \cdot \nabla_{\mathbf{x}} \mathbf{u}^{0}+\frac{\mathcal{P}^{\prime \prime}\left(\rho_{0}\right)}{\rho_{0}} r^{0} \nabla_{\mathbf{x}} r^{0}+\frac{\mathcal{P}^{\prime}\left(\rho_{0}\right)}{\rho_{0}} \nabla_{\mathbf{y}} r^{0}-\frac{\mu}{\rho_{0}} \Delta_{\mathbf{x}} \mathbf{u}^{0}-\frac{\mu+\lambda}{\rho_{0}} \nabla_{\mathbf{x}} \nabla_{\mathbf{x}} \cdot \mathbf{u}^{0}
\end{array}\right)
$$

as the equation satisfied by the limit solution $\left(r^{0}, \mathbf{u}^{0}\right)$.

\subsection{Calculation of the projection operator}

In order to write equation (4.15) more explicitly, note that the zero eigenspace of $\mathbb{L}$ is spanned by the $\mathrm{x}$-independent density components and the divergence-free velocity vectors. The restriction of $\mathbb{S}$ to that subspace is the identity operator, so by (4.12) the restriction of $\mathbb{E}$ to that subspace is simply $\mathbb{M}_{\tau}$. Since this part of $\mathbb{E}$ projects onto functions that do not depend on the fast time $\tau$, it is called the slow part of $\mathbb{E}$. The slow part 
of $\mathbb{E}$ is thus the projection onto functions $r$ that are independent of $\mathbf{x}$ and $\tau$ and onto functions $\mathbf{u}$ that are independent of $\tau$ and divergence-free:

$$
\mathbb{E}_{\text {slow }}=\left(\begin{array}{cc}
\mathbb{M}_{\tau, \mathbf{x}} & 0 \\
0 & \mathbb{M}_{\tau} \mathbb{P}_{\mathbf{x}}
\end{array}\right)
$$

where $\mathbb{M}_{z}$ denotes the average with respect to the variables $z$ and $\mathbb{P}_{\mathbf{x}} w:=w-\nabla_{\mathbf{x}} \Delta_{\mathbf{x}}^{-1} \nabla_{\mathbf{x}} \cdot w$ is the usual projection onto divergence-free vector fields.

In order to calculate the "fast" part of $\mathbb{E}$ that projects onto functions that do depend on $\tau$, return to the Fourier-space representation of the solution operator $\mathbb{S}$ in (4.8), and note that for each nonzero $\mathbf{k}$ the matrix $\widehat{\mathbb{L}}(\mathbf{k}):=\mathbb{F}_{\mathbf{x}} \mathbb{L F}_{\mathbf{x}}^{-1}$ has exactly two nonzero eigenvalues. The union of the corresponding eigenvectors over all $\mathbf{k}$ spans a "fast" space on which the solution to (4.7) truly depends on $\tau$. Those nonzero eigenvalues and corresponding eigenvectors $\mathbf{w}_{(\mathbf{k})}^{( \pm)}$are

$$
\widehat{\mathbb{L}} \mathbf{w}_{(\mathbf{k})}^{( \pm)}=\left(\begin{array}{cc}
0 & -\rho_{0} i \mathbf{k} \cdot \\
-\frac{\mathcal{P}^{\prime}\left(\rho_{0}\right)}{\rho_{0}} i \mathbf{k} & 0
\end{array}\right)\left(\begin{array}{c}
\mp \frac{i \rho_{0}|\mathbf{k}|}{\sqrt{\mathcal{P}^{\prime}\left(\rho_{0}\right)}} \\
i \mathbf{k}
\end{array}\right)= \pm i \sqrt{\mathcal{P}^{\prime}\left(\rho_{0}\right)}|\mathbf{k}|\left(\begin{array}{c}
\mp \frac{i \rho_{0}|\mathbf{k}|}{\sqrt{\mathcal{P}^{\prime}\left(\rho_{0}\right)}} \\
i \mathbf{k}
\end{array}\right) .
$$

Restricted to the span of those eigenvectors, the projection $\mathbb{E}$ singles out those functions of the form

$$
c^{( \pm)}(t, \mathbf{k}, \mathbf{y}) e^{ \pm i \sqrt{\mathcal{P}^{\prime}\left(\rho_{0}\right)}|\mathbf{k}| \tau} \mathbf{w}_{(\mathbf{k})}^{( \pm)} .
$$

Note that for each $\mathbf{k}$ the two vectors $\mathbf{w}_{(\mathbf{k})}^{( \pm)}$are orthogonal with respect to the inner product $\mathbf{g}^{T} \tilde{A} \mathbf{h}$. Also, for functions $r^{0}$ having the $\tau$-dependence indicated in (4.18), $\mp \frac{i \rho_{0}}{\sqrt{\mathcal{P}^{\prime}\left(\rho_{0}\right)}}|\mathbf{k}| r^{0}=-\frac{\rho_{0}}{\mathcal{P}^{\prime}\left(\rho_{0}\right)} \partial_{\tau} r^{0}$. Hence the fast part of the projection $\mathbb{E}$ is

$$
\begin{aligned}
& \mathbb{E}_{\text {fast }}=\mathbb{F}_{\mathbf{x}}^{-1}\left\{\sum_{ \pm} \frac{1}{\left(w_{(\mathbf{k})}^{( \pm)}\right)^{T} \tilde{A} w_{(\mathbf{k})}^{( \pm)}} w_{(\mathbf{k})}^{( \pm)} \mathrm{e}^{ \pm i \sqrt{\mathcal{P}^{\prime}\left(\rho_{0}\right)}|\mathbf{k}| \tau} \mathbb{M}_{\tau}\left[\mathrm{e}^{\mp i \sqrt{\mathcal{P}^{\prime}\left(\rho_{0}\right)}|\mathbf{k}| \tau}\left(w_{(\mathbf{k})}^{( \pm)}\right)^{T} \tilde{A} \mathbb{F}_{\mathbf{x}}\left(I-\mathbb{M}_{\mathbf{x}}\right) \cdot\right]\right\}
\end{aligned}
$$

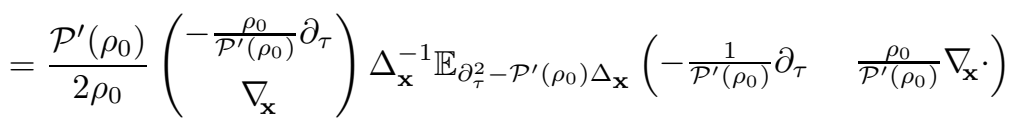

$$
\begin{aligned}
& =\frac{1}{2}\left(\begin{array}{c}
-\frac{\rho_{0}}{\mathcal{P}^{\prime}\left(\rho_{0}\right)} \partial_{\tau} \\
\nabla_{\mathbf{x}}
\end{array}\right) \Delta_{\mathbf{x}}^{-1} \mathbb{E}_{\partial_{\tau}^{2}-\mathcal{P}^{\prime}\left(\rho_{0}\right) \Delta_{\mathbf{x}}}\left(\begin{array}{cc}
-\frac{1}{\rho_{0}} \partial_{\tau} & \left.\nabla_{\mathbf{x}} \cdot\right)
\end{array}\right),
\end{aligned}
$$

where $\mathbb{E}_{\partial_{\tau}^{2}-\mathcal{P}^{\prime}\left(\rho_{0}\right) \Delta_{\mathbf{X}}}$ denotes the projection onto solutions of the scalar wave equation $\left[\partial_{\tau}^{2}-\mathcal{P}^{\prime}\left(\rho_{0}\right) \Delta_{\mathbf{x}}\right] w=0$, i.e.,

$$
\mathbb{F}_{\mathbf{x}} \mathbb{E}_{\partial_{\tau}^{2}-\mathcal{P}^{\prime}\left(\rho_{0}\right) \Delta_{\mathbf{x}}} \mathbb{F}_{\mathbf{x}}^{-1}=\sum_{ \pm} \mathrm{e}^{ \pm i \sqrt{\mathcal{P}^{\prime}\left(\rho_{0}\right)}|\mathbf{k}| \tau} \mathbb{M}_{\tau}\left[\mathrm{e}^{\mp i \sqrt{\mathcal{P}^{\prime}\left(\rho_{0}\right)}|\mathbf{k}| \tau} \cdot\right]
$$

Note that the projection $I-\mathbb{M}_{\mathbf{x}}$ is not needed in the later formulas in (4.19) since applying the operators there in the order indicated annihilates the $\mathbf{x}$-independent functions. To verify formula (4.19), note that it annihilates constant densities $r$ and divergence-free velocities $\mathbf{u}$, and when restricted to the span of the $\mathbf{w}_{(\mathbf{k})}^{( \pm)}$it reproduces functions of the form (4.18) but annihilates functions having different $\tau$-dependence.

\subsection{Explicit limit equations}

\subsubsection{Separation into fast and slow parts}

The formulas just derived allow us to write the limit equations (4.15) more explicitly. Since the slow and fast projections are mutually orthogonal, (4.15) remains valid when the full projection $\mathbb{E}$ is replaced by either its fast 
or slow part. Similarly, (4.14) implies that $\mathbf{v}^{0}$ equals the sum $\mathbf{v}_{\text {fast }}^{0}+\mathbf{v}_{\text {slow }}^{0}$ of its fast and slow projections, i.e.

$$
\left(\begin{array}{c}
r^{0} \\
\mathbf{u}^{0}
\end{array}\right)=\left(\begin{array}{c}
r_{\text {fast }}^{0} \\
\mathbf{u}_{\text {fast }}^{0}
\end{array}\right)+\left(\begin{array}{c}
r_{\text {slow }}^{0} \\
\mathbf{u}_{\text {slow }}^{0}
\end{array}\right) .
$$

By (4.19) the fast part satisfies

$$
\mathbb{M}_{\tau}\left(\begin{array}{c}
r_{\text {fast }}^{0} \\
\mathbf{u}_{\text {fast }}^{0}
\end{array}\right)=\left(\begin{array}{l}
0 \\
0
\end{array}\right)
$$

and

$$
\left(\begin{array}{c}
r_{\text {fast }}^{0} \\
\mathbf{u}_{\text {fast }}^{0}
\end{array}\right)=\left(\begin{array}{c}
-\frac{\rho_{0}}{\mathcal{P}^{\prime}\left(\rho_{0}\right)} \partial_{\tau} \\
\nabla_{\mathbf{x}}
\end{array}\right) \phi^{0},
$$

where

$$
\phi^{0}:=\frac{1}{2} \Delta_{\mathbf{x}}^{-1}\left(\nabla_{\mathbf{x}} \cdot \mathbf{u}^{0}-\frac{1}{\rho_{0}} r_{\tau}^{0}\right)
$$

satisfies the wave equation

$$
\left[\partial_{\tau}^{2}-\mathcal{P}^{\prime}\left(\rho_{0}\right) \Delta_{\mathbf{x}}\right] \phi^{0}=0 .
$$

By (4.16), the slow part satisfies

$$
r_{\text {slow }}^{0}=\mathbb{M}_{\mathbf{x}} r_{\text {slow }}^{0}=r_{\text {slow }}^{0}(t, \mathbf{y}), \quad \mathbf{u}_{\text {slow }}^{0}=\mathbf{u}_{\text {slow }}^{0}(t, \mathbf{x}, \mathbf{y}), \quad \nabla_{\mathbf{x}} \cdot \mathbf{u}_{\text {slow }}^{0}=0 .
$$

In addition, (4.23) implies that

$$
\mathbb{P}_{\mathbf{x}}\left[\mathbf{u}_{\text {fast }}^{0} \cdot \nabla_{\mathbf{x}} \mathbf{u}_{\text {fast }}^{0}\right]=\mathbb{P}_{\mathbf{x}}\left[\nabla_{\mathbf{x}} \phi^{0} \cdot \nabla_{\mathbf{x}}\left(\nabla_{\mathbf{x}} \phi^{0}\right)\right]=\frac{1}{2} \mathbb{P}_{\mathbf{x}}\left[\nabla_{\mathbf{x}}\left|\nabla_{\mathbf{x}} \phi^{0}\right|^{2}\right]=0,
$$

and (4.22) and (4.26) imply that

$$
\mathbb{M}_{\tau}\left[w_{\text {fast }} w_{\text {slow }}\right]=0
$$

for any fast $w_{\text {fast }}$ and slow $w_{\text {slow }}$.

\subsubsection{The slow equations}

Replacing the full projection $\mathbb{E}$ in (4.15) by its slow part (4.16) and using (4.3), (4.21), (4.22), (4.23), (4.26), (4.27), and (4.28) yields

$$
\begin{aligned}
& \left(\begin{array}{l}
0 \\
0
\end{array}\right)=\mathbb{E}_{\text {slow }}\left(\begin{array}{c}
r_{t}^{0}+\mathbf{u}^{0} \cdot \nabla_{\mathbf{x}} r^{0}+r^{0} \nabla_{\mathbf{x}} \cdot \mathbf{u}^{0}+\rho_{0} \nabla_{\mathbf{y}} \cdot \mathbf{u}^{0} \\
\frac{r^{0}}{\rho_{0}} u_{\tau}^{0}+\mathbf{u}_{t}^{0}+\mathbf{u}^{0} \cdot \nabla_{\mathbf{x}} \mathbf{u}^{0}+\frac{\mathcal{P}^{\prime \prime}\left(\rho_{0}\right)}{\rho_{0}} r^{0} \nabla_{\mathbf{x}} r^{0}+\frac{\mathcal{P}^{\prime}\left(\rho_{0}\right)}{\rho_{0}} \nabla_{\mathbf{y}} r^{0}-\frac{\mu}{\rho_{0}} \Delta_{\mathbf{x}} \mathbf{u}^{0}-\frac{\mu+\lambda}{\rho_{0}} \nabla_{\mathbf{x}} \nabla_{\mathbf{x}} \cdot \mathbf{u}^{0}
\end{array}\right) \\
& =\partial_{t}\left(\begin{array}{c}
r_{\text {slow }}^{0} \\
\mathbf{u}_{\text {slow }}^{0}
\end{array}\right)+\left(\begin{array}{c}
\mathbb{M}_{\tau, \mathbf{x}}\left[\nabla_{\mathbf{x}} \cdot\left[r^{0} \mathbf{u}^{0}\right]+\rho_{0} \nabla_{\mathbf{y}} \cdot \mathbf{u}^{0}\right] \\
\mathbb{M}_{\tau} \mathbb{P}_{\mathbf{x}}\left[\nabla_{\mathbf{x}}\left\{\frac{1}{2}\left[\frac{\mathcal{P}^{\prime \prime}\left(\rho_{0}\right)}{\rho_{0}}-\frac{\mathcal{P}^{\prime}\left(\rho_{0}\right)}{\rho_{0}^{2}}\right]\left(r^{0}\right)^{2}\right\}+\left[\mathbf{u}^{0} \cdot \nabla_{\mathbf{x}} \mathbf{u}^{0}\right]+\frac{\mathcal{P}^{\prime}\left(\rho_{0}\right)}{\rho_{0}} \nabla_{\mathbf{y}} r^{0}-\frac{\mu}{\rho_{0}} \Delta_{\mathbf{x}} \mathbf{u}^{0}\right]
\end{array}\right) \\
& =\partial_{t}\left(\begin{array}{c}
r_{\text {slow }}^{0} \\
\mathbf{u}_{\text {slow }}^{0}
\end{array}\right)+\left(\begin{array}{c}
\rho_{0} \nabla_{\mathbf{y}} \cdot \mathbb{M}_{\mathbf{x}} \mathbf{u}_{\text {slow }}^{0} \\
\mathbb{M}_{\tau} \mathbb{P}_{\mathbf{x}}\left[\left(\mathbf{u}_{\text {slow }}^{0}+\nabla_{\mathbf{x}} \phi^{0}\right) \cdot \nabla_{\mathbf{x}}\left(\mathbf{u}_{\text {slow }}^{0}+\nabla_{\mathbf{x}} \phi^{0}\right)\right]+\frac{\mathcal{P}^{\prime}\left(\rho_{0}\right)}{\rho_{0}} \nabla_{\mathbf{y}} r_{\text {slow }}^{0}-\frac{\mu}{\rho_{0}} \Delta_{\mathbf{x}} \mathbf{u}_{\text {slow }}^{0}
\end{array}\right) \\
& =\partial_{t}\left(\begin{array}{c}
r_{\text {slow }}^{0} \\
\mathbf{u}_{\text {slow }}^{0}
\end{array}\right)+\left(\begin{array}{c}
\rho_{0} \nabla_{\mathbf{y}} \cdot \mathbb{M}_{\mathbf{x}} \mathbf{u}_{\text {slow }}^{0} \\
\mathbb{P}_{\mathbf{x}}\left[\mathbf{u}_{\text {slow }}^{0} \cdot \nabla_{\mathbf{x}} \mathbf{u}_{\text {slow }}^{0}\right]+\frac{\mathcal{P}^{\prime}\left(\rho_{0}\right)}{\rho_{0}} \nabla_{\mathbf{y}} r_{\text {slow }}^{0}-\frac{\mu}{\rho_{0}} \Delta_{\mathbf{x}} \mathbf{u}_{\text {slow }}^{0}
\end{array}\right) .
\end{aligned}
$$

Note that when the initial data, and hence also the solution, have no dependence on $\mathbf{y}$ then the second equation of (4.29) plus the last part of (4.26) yield the standard incompressible Navier-Stokes equations. In particular, 
this holds even though the zeroth-order solution may have a nonzero fast part. In fact, even when $\mathbf{y}$-dependence is present the zeroth-order slow equations are independent of the fast part of the solution.

\subsubsection{The fast equation}

As noted in (4.23), the fast part of the zeroth-order solution is determined uniquely by $\phi^{0}$, so it suffices to derive an equation for the latter. Moreover, (4.15) with $\mathbb{E}$ replaced by its fast part (4.19) reduces to a scalar equation, which can be simplified by using (4.24)-(4.25), (4.3), (4.21), (4.23), (4.26), (4.27), and (4.28):

$$
\begin{aligned}
0= & \frac{1}{2} \Delta_{\mathbf{x}}^{-1} \mathbb{E}_{\partial_{\tau}^{2}-\mathcal{P}^{\prime}\left(\rho_{0}\right) \Delta_{\mathbf{x}}}\left(-\frac{1}{\rho_{0}} \partial_{\tau} \quad \nabla_{\mathbf{x}} \cdot\right) \cdot \\
& \left(\frac{r^{0}}{\rho_{0}} u_{\tau}^{0}+\mathbf{u}_{t}^{0}+\mathbf{u}^{0} \cdot \nabla_{\mathbf{x}} \mathbf{u}^{0}+\frac{\mathcal{P}^{\prime \prime}\left(\rho_{0}\right)}{\rho_{0}} r^{0} \nabla_{\mathbf{x}} r^{0}+\frac{\mathcal{P}^{\prime}\left(\rho_{0}\right)}{\rho_{0}} \nabla_{\mathbf{y}} r^{0}-\frac{\mu}{\rho_{0}} \Delta_{\mathbf{x}} \mathbf{u}^{0}-\frac{\mu+\lambda}{\rho_{0}} \nabla_{\mathbf{x}} \nabla_{\mathbf{x}} \cdot \mathbf{u}^{0}\right) \\
= & \phi_{t}^{0}+\frac{1}{2} \Delta_{\mathbf{x}}^{-1} \mathbb{E}_{\partial_{\tau}^{2}-\mathcal{P}^{\prime}\left(\rho_{0}\right) \Delta_{\mathbf{x}}}\left\{-\frac{1}{\rho_{0}} \partial_{\tau}\left[\mathbf{u}^{0} \cdot \nabla_{\mathbf{x}} r_{\text {fast }}^{0}+r^{0} \nabla_{\mathbf{x}} \cdot \mathbf{u}_{\text {fast }}^{0}+\rho_{0} \nabla_{\mathbf{y}} \cdot \mathbf{u}^{0}\right]\right. \\
& \left.+\nabla_{\mathbf{x}} \cdot\left[\mathbf{u}^{0} \cdot \nabla_{\mathbf{x}} \mathbf{u}^{0}+\frac{1}{2}\left(\frac{\mathcal{P}^{\prime \prime}\left(\rho_{0}\right)}{\rho_{0}}-\frac{\mathcal{P}^{\prime}\left(\rho_{0}\right)}{\rho_{0}^{2}}\right) \nabla_{\mathbf{x}}\left(r^{0}\right)^{2}+\frac{\mathcal{P}^{\prime}\left(\rho_{0}\right)}{\rho_{0}} \nabla_{\mathbf{y}} r^{0}-\frac{\mu}{\rho_{0}} \Delta_{\mathbf{x}} \mathbf{u}^{0}-\frac{\mu+\lambda}{\rho_{0}} \nabla_{\mathbf{x}} \nabla_{\mathbf{x}} \cdot \mathbf{u}_{\text {fast }}^{0}\right]\right\} \\
= & \phi_{t}^{0}+\frac{1}{2} \Delta_{\mathbf{x}}^{-1} \mathbb{E}_{\partial_{\tau}^{2}-\mathcal{P}^{\prime}\left(\rho_{0}\right) \Delta_{\mathbf{x}}}\left\{-\frac{1}{\rho_{0}} \partial_{\tau}\left[\mathbf{u}^{0} \cdot \nabla_{\mathbf{x}} r_{\text {fast }}^{0}+r^{0} \nabla_{\mathbf{x}} \cdot \mathbf{u}_{\text {fast }}^{0}\right]+\nabla_{\mathbf{y}} \cdot\left[-\mathbf{u}_{\tau}^{0}+\frac{\mathcal{P}^{\prime}\left(\rho_{0}\right)}{\rho_{0}} \nabla_{\mathbf{x}} r^{0}\right]\right. \\
& \left.+\Delta_{\mathbf{x}}\left[\frac{1}{2}\left(\frac{\mathcal{P}^{\prime \prime}\left(\rho_{0}\right)}{\rho_{0}}-\frac{\mathcal{P}^{\prime}\left(\rho_{0}\right)}{\rho_{0}^{2}}\right)\left[\left(r_{\text {fast }}^{0}\right)^{2}+2 r_{\text {slow }}^{0} r_{\text {fast }}^{0}\right]-\frac{2 \mu+\lambda}{\rho_{0}} \nabla_{\mathbf{x}} \cdot \mathbf{u}_{\text {fast }}^{0}\right]+\nabla_{\mathbf{x}} \cdot\left[\mathbf{u}^{0} \cdot \nabla_{\mathbf{x}} \mathbf{u}^{0}\right]\right\} \\
= & \phi_{t}^{0}+\frac{1}{2} \Delta_{\mathbf{x}}^{-1} \mathbb{E}_{\partial_{\tau}^{2}-\mathcal{P}^{\prime}\left(\rho_{0}\right) \Delta_{\mathbf{x}}}\left\{-\frac{1}{\rho_{0}} \partial_{\tau}\left[-\frac{\rho_{0}}{\mathcal{P}^{\prime}\left(\rho_{0}\right)}\left(\mathbf{u}_{\text {slow }}^{0}+\nabla_{\mathbf{x}} \phi^{0}\right) \cdot \nabla_{\mathbf{x}} \phi_{\tau}^{0}+\left(r_{\text {slow }}^{0}-\frac{\rho_{0}}{\mathcal{P}^{\prime}\left(\rho_{0}\right)} \phi_{\tau}^{0}\right) \Delta_{\mathbf{x}} \phi^{0}\right]\right. \\
& +\Delta_{\mathbf{x}}\left[\frac{1}{2}\left(\frac{\mathcal{P}^{\prime \prime}\left(\rho_{0}\right)}{\rho_{0}}-\frac{\mathcal{P}^{\prime}\left(\rho_{0}\right)}{\rho_{0}^{2}}\right)\left[\left(\frac{\rho_{0}}{\mathcal{P}^{\prime}\left(\rho_{0}\right)}\right)^{2}\left(\phi_{\tau}^{0}\right)^{2}-\frac{2 \rho_{0}}{\mathcal{P}^{\prime}\left(\rho_{0}\right)} r_{\text {slow }}^{0} \phi_{\tau}^{0}\right]-\frac{2 \mu+\lambda}{\rho_{0}} \Delta_{\mathbf{x}} \phi^{0}\right] \\
& \left.+\nabla_{\mathbf{x}} \cdot\left[\frac{1}{2} \nabla_{\mathbf{x}}\left|\nabla_{\mathbf{x}} \phi^{0}\right|^{2}+\left(\mathbf{u}_{\text {slow }}^{0} \cdot \nabla_{\mathbf{x}}\right) \nabla_{\mathbf{x}} \phi^{0}+\left(\nabla_{\mathbf{x}} \phi^{0} \cdot \nabla_{\mathbf{x}}\right) \mathbf{u}_{\text {slow }}^{0}-2 \nabla_{\mathbf{y}} \phi_{\tau}^{0}\right]\right\} \cdot
\end{aligned}
$$

To simplify (4.30) further, note that by (4.26) and (4.25),

$$
-\frac{1}{\rho_{0}} \partial_{\tau}\left[-\frac{\rho_{0}}{\mathcal{P}^{\prime}\left(\rho_{0}\right)} \mathbf{u}_{\text {slow }}^{0} \cdot \nabla_{\mathbf{x}} \phi_{\tau}^{0}\right]=\mathbf{u}_{\text {slow }}^{0} \cdot \nabla_{\mathbf{x}} \frac{1}{\mathcal{P}^{\prime}\left(\rho_{0}\right)} \partial_{\tau}^{2} \phi^{0}=\mathbf{u}_{\text {slow }}^{0} \cdot \nabla_{\mathbf{x}} \Delta_{\mathbf{x}} \phi^{0},
$$

and combining this with the term $\nabla_{\mathbf{x}} \cdot\left[\left(\nabla_{\mathbf{x}} \phi^{0} \cdot \nabla_{\mathbf{x}}\right) \mathbf{u}_{\text {slow }}^{0}\right]$ from (4.30) yields another copy of the term

$$
\nabla_{\mathbf{x}} \cdot\left[\left(\mathbf{u}_{\text {slow }}^{0} \cdot \nabla_{\mathbf{x}}\right) \nabla_{\mathbf{x}} \phi^{0}\right]
$$

already present in (4.30), because $\mathbf{u}_{\text {slow }}^{0}$ is divergence-free. Similarly,

$$
\begin{aligned}
-\frac{1}{\rho_{0}} \partial_{\tau} & {\left[-\frac{\rho_{0}}{\mathcal{P}^{\prime}\left(\rho_{0}\right)} \nabla_{\mathbf{x}} \phi^{0} \cdot \nabla_{\mathbf{x}} \phi_{\tau}^{0}+\left(r_{\text {slow }}^{0}-\frac{\rho_{0}}{\mathcal{P}^{\prime}\left(\rho_{0}\right)} \phi_{\tau}^{0}\right) \Delta_{\mathbf{x}} \phi^{0}\right] } \\
& =\frac{1}{\mathcal{P}^{\prime}\left(\rho_{0}\right)} \partial_{\tau} \nabla_{\mathbf{x}} \cdot\left[\phi_{\tau}^{0} \nabla_{\mathbf{x}} \phi^{0}\right]-\Delta_{\mathbf{x}}\left[\frac{r_{\text {slow }}^{0}}{\rho_{0}} \phi_{\tau}^{0}\right] \\
& =\nabla_{\mathbf{x}} \cdot\left[\Delta_{\mathbf{x}} \phi^{0} \nabla_{\mathbf{x}} \phi^{0}\right]+\Delta_{\mathbf{x}}\left[\frac{1}{2 \mathcal{P}^{\prime}\left(\rho_{0}\right)}\left(\phi_{\tau}^{0}\right)^{2}\right]-\Delta_{\mathbf{x}}\left[\frac{r_{\text {slow }}^{0}}{\rho_{0}} \phi_{\tau}^{0}\right] .
\end{aligned}
$$


Since the terms involving $\Delta_{\mathbf{x}}$ in the final expression in (4.32) cancel terms already present in (4.30), substituting (4.31) and (4.32) into (4.30) and noting that $\Delta_{\mathbf{x}}^{-1} \Delta_{\mathbf{x}}=I-\mathbb{M}_{\mathbf{X}}$ and that the projection $\mathbb{E}_{\partial_{\tau}^{2}-\mathcal{P}^{\prime}\left(\rho_{0}\right) \Delta_{\mathbf{X}}}$ is superfluous when applied to terms linear in $\phi^{0}$ with coefficients either constant or at most depending on $r_{\text {slow }}^{0}$ yields

$$
\begin{aligned}
0= & \phi_{t}^{0}-\frac{\mathcal{P}^{\prime \prime}\left(\rho_{0}\right)}{\mathcal{P}^{\prime}\left(\rho_{0}\right)} r_{\text {slow }}^{0} \phi_{\tau}^{0}-\frac{2 \mu+\lambda}{\rho_{0}} \Delta_{\mathbf{x}} \phi^{0}+\frac{1}{4} \mathbb{E}_{\partial_{\tau}^{2}-\mathcal{P}^{\prime}\left(\rho_{0}\right) \Delta_{\mathbf{x}}}\left(1-\mathbb{M}_{\mathbf{x}}\right)\left[\frac{\mathcal{P}^{\prime \prime}\left(\rho_{0}\right) \rho_{0}}{\mathcal{P}^{\prime}\left(\rho_{0}\right)^{2}}\left(\phi_{\tau}^{0}\right)^{2}+\left|\nabla_{\mathbf{x}} \phi^{0}\right|^{2}\right] \\
& +\Delta_{\mathbf{x}}^{-1} \mathbb{E}_{\partial_{\tau}^{2}-\mathcal{P}^{\prime}\left(\rho_{0}\right) \Delta_{\mathbf{x}}}\left\{\nabla_{\mathbf{x}} \cdot\left[\frac{1}{2} \Delta_{\mathbf{x}} \phi^{0} \nabla_{\mathbf{x}} \phi^{0}+\left(\mathbf{u}_{\text {slow }}^{0} \cdot \nabla_{\mathbf{x}}\right) \nabla_{\mathbf{x}} \phi^{0}\right]\right\}-\Delta_{\mathbf{x}}^{-1} \nabla_{\mathbf{x}} \cdot \nabla_{\mathbf{y}} \phi_{\tau}^{0} .
\end{aligned}
$$

\subsubsection{Average over smaller spatial scale}

Whenever several spatial scales are present in a single problem it is of interest to consider how motions on the different scales interact. More specifically, the question is whether small-scale motions produce large-scale effects. Now (4.23) plus (4.24) show that average $\mathbb{M}_{\mathbf{x}}$ over the small scale variable $\mathbf{x}$ of the fast part of the lowest-order solution vanishes identically. As for the slow part, since the equation $\nabla_{\mathbf{x}} \cdot \mathbf{u}_{\text {slow }}^{0}=0$ in (4.26) implies that the term $\mathbf{u}_{\text {slow }}^{0} \cdot \nabla_{\mathbf{x}} \mathbf{u}_{\text {slow }}^{0}$ in (4.29) is an $\mathbf{x}$ derivative, applying $\mathbb{M}_{\mathbf{x}}$ to the slow equations (4.29) yields the linear acoustics equations

$$
\partial_{t}\left(\begin{array}{c}
\mathbb{M}_{\mathbf{x}} r_{\text {slow }}^{0} \\
\mathbb{M}_{\mathbf{x}} \mathbf{u}_{\text {slow }}^{0}
\end{array}\right)+\left(\begin{array}{c}
\rho_{0} \nabla_{\mathbf{y}} \mathbb{M}_{\mathbf{x}} \mathbf{u}_{\text {slow }}^{0} \\
\frac{\mathcal{P}^{\prime}\left(\rho_{0}\right)}{\rho_{0}} \nabla_{\mathbf{y}} \mathbb{M}_{\mathbf{x}} r_{\text {slow }}^{0}
\end{array}\right)=\left(\begin{array}{l}
0 \\
0
\end{array}\right),
$$

for the average over the faster spatial scale, which shows that the small-scale motion does not affect the largescale flow.

\section{THE CONVERGENCE THEOREM AND REMARKS ON ITS PROOF}

The equations to which the convergence theorem applies are obtained from the scaled barotropic, possibly viscous, equations (3.13)-(3.14) by omitting the derivatives with respect to the additional time variable $\tau$, i.e.,

$$
r_{t}+\mathbf{u} \cdot \nabla_{\mathbf{x}} r+\varepsilon \mathbf{u} \cdot \nabla_{\mathbf{y}} r+\frac{\left(\rho_{0}+\varepsilon r\right)}{\varepsilon} \nabla_{\mathbf{x}} \cdot \mathbf{u}+\left(\rho_{0}+\varepsilon r\right) \nabla_{\mathbf{y}} \cdot \mathbf{u}=0
$$

and

$$
\begin{aligned}
& \left(\rho_{0}+\varepsilon r\right)\left[\mathbf{u}_{t}+\mathbf{u} \cdot \nabla_{\mathbf{x}} \mathbf{u}+\varepsilon \mathbf{u} \cdot \nabla_{\mathbf{y}} \mathbf{u}\right]+\frac{\mathcal{P}^{\prime}\left(\rho_{0}+\varepsilon r\right)}{\varepsilon} \nabla_{\mathbf{x}} r+\mathcal{P}^{\prime}\left(\rho_{0}+\varepsilon r\right) \nabla_{\mathbf{y}} r \\
& =\mu\left[\Delta_{\mathbf{x}} \mathbf{u}+2 \varepsilon \nabla_{\mathbf{x}} \cdot \nabla_{\mathbf{y}} \mathbf{u}+\varepsilon^{2} \Delta_{\mathbf{y}} \mathbf{u}\right]+(\mu+\lambda)\left[\nabla_{\mathbf{x}} \nabla_{\mathbf{x}} \cdot \mathbf{u}+\varepsilon\left(\nabla_{\mathbf{x}} \nabla_{\mathbf{y}} \cdot \mathbf{u}+\nabla_{\mathbf{y}} \nabla_{\mathbf{x}} \cdot \mathbf{u}\right)+\varepsilon^{2} \nabla_{\mathbf{y}} \nabla_{\mathbf{y}} \cdot \mathbf{u}\right] .
\end{aligned}
$$

This does not mean that the $\tau$ time scale is not present in the solution but only that since the solution of equations (3.13)-(3.14) depends on $\varepsilon$ directly, not just through the variables $y$ and $\tau$, those equations do not truly determine the division of the time-dependence between the two variables $t$ and $\tau$. It is therefore necessary either to add some condition that will determine the $\tau$ dependence or, as done in (5.1)-(5.2), to lump all the time-dependence into a single time variable. This problem does not arise for the multiple spatial variables because it can be assumed that the division of the spatial dependence into dependence on the two variables $\mathbf{x}$ and $\mathbf{y}$ has been performed on the initial data.

The case for which the asymptotics derived above apply most directly is when the initial data, and hence also the solution, are periodic in the small-scale spatial variable $\mathbf{x}$ :

Theorem 5.1. Suppose that the initial data $\left(r_{0, \varepsilon}(\mathbf{x}, \mathbf{y}), \mathbf{u}_{0, \varepsilon}(\mathbf{x}, \mathbf{y})\right)$ for (5.1)-(5.2) are periodic in $\mathbf{x}$ and either periodic in $\mathbf{y}$ or defined for that variable in the whole space $\mathbb{R}^{d}$, are uniformly bounded in $H^{s}$ for $s$ sufficiently 
large, and converge in $H^{s}$ as $\varepsilon \rightarrow 0$ to $\left(r_{0,0}(\mathbf{x}, \mathbf{y}), \mathbf{u}_{0,0}(\mathbf{x}, \mathbf{y})\right)$. Then the corresponding solution to (5.1)-(5.2) exists for a time independent of $\varepsilon$, and

$$
\left(\begin{array}{l}
r_{\varepsilon}(t, \mathbf{x}, \mathbf{y}) \\
\mathbf{u}_{\varepsilon}(t, \mathbf{x}, \mathbf{y})
\end{array}\right)-\left.\left(\begin{array}{l}
r^{0}(t, \tau, \mathbf{x}, \mathbf{y}) \\
\mathbf{u}^{0}(t, \tau, \mathbf{x}, \mathbf{y})
\end{array}\right)\right|_{\tau=\frac{t}{\varepsilon}} \rightarrow 0
$$

in $H_{\text {loc }}^{s-\delta}$ as $\varepsilon \rightarrow 0$ for any $\delta>0$, where the "limit profile" $\left(\begin{array}{l}r^{0} \\ \mathbf{u}^{0}\end{array}\right)$ is the sum (4.21) of slow and fast parts that are the solutions of (4.29) and (4.23), (4.25), and (4.33), respectively, with initial data

$$
\mathbf{u}_{\text {slow }}^{0}(0, \mathbf{x}, \mathbf{y})=\mathbb{P}_{\mathbf{x}} \mathbf{u}_{0,0}(\mathbf{x}, \mathbf{y}), \quad r_{\text {slow }}^{0}(0, \mathbf{y})=\mathbb{M}_{\mathbf{x}} r_{0,0}(\mathbf{x}, \mathbf{y})
$$

and

$$
\phi^{0}(0,0, \mathbf{x}, \mathbf{y})=\Delta_{\mathbf{x}}^{-1} \nabla_{\mathbf{x}} \cdot \mathbf{u}_{0,0}(\mathbf{x}, \mathbf{y}), \quad \phi_{\tau}^{0}(0,0, \mathbf{x}, \mathbf{y})=-\frac{\mathcal{P}^{\prime}\left(\rho_{0}\right)}{\rho_{0}}\left(I-\mathbb{M}_{\mathbf{x}}\right) r_{0,0}(\mathbf{x}, \mathbf{y}) .
$$

For the relevant values of $s$, convergence in $H_{\text {loc }}^{s-\delta}$ implies convergence in $C^{2}$, and the convergence of (5.3) in the latter space remains valid when $\mathbf{y}$ is set equal to $\varepsilon \mathbf{x}$ as is done in the transformation (3.11) from the original equations (3.5) and (1.2) to the scaled equations (5.1)-(5.2). Theorem 5.1 therefore implies an analogous result in terms of the solution of the original equations.

Theorem 5.1 follows from the general convergence theorem for singular perturbations in [25]. An alternative proof and explicit calculation of the limit with only one spatial scale was given in [44]. Although only the inviscid case was treated there, adding viscosity in an essentially diagonal form as in (5.1)-(5.2) causes no problems for smooth solutions in domains without boundaries, except that the proof may require the initial data to have one more derivative since the smoothing effect of the viscous terms is not made use of in the proof.

There are two general approaches for proving "convergence" results like (5.3) for singular perturbation problems: in the direct approach one first obtains estimates for solutions $\mathbf{v}^{0}$ of the limit problem and/or uniform estimates for the solutions $\mathbf{v}^{\varepsilon}$ of the full problem, and then estimates the difference between the two [25]. Alternatively, in the compactness method one obtains uniform estimates for both $\mathbf{v}^{\varepsilon}$ and $\partial_{t} \mathbf{v}^{\varepsilon}$, then applies Ascoli's theorem or some extension thereof to obtain convergence to a limit for a sequence of values of $\varepsilon$, and finally shows uniqueness of the limit $\mathbf{v}^{0}$ to obtain convergence without restriction to a sequence [44].

To see how the compactness approach works for system (5.1)-(5.2), note that after multiplying the first equation by $\frac{\mathcal{P}^{\prime}\left(\rho_{0}+\varepsilon r\right)}{\rho_{0}+\varepsilon r}$ those equations take the form

$$
A_{0}(\varepsilon \mathbf{v}) \mathbf{v}_{t}+\sum_{j} A_{j}(\mathbf{v}, \varepsilon) \mathbf{v}_{x_{j}}+\sum_{j} B_{j}(\mathbf{v}, \varepsilon) \mathbf{v}_{y_{j}}+\frac{1}{\varepsilon} \sum_{j} C_{j} \mathbf{v}_{x_{j}}=\sum_{m \leq n} K_{m, n}\left[\partial_{x_{m}}+\varepsilon \partial_{y_{m}}\right]\left[\partial_{x_{n}}+\varepsilon \partial_{y_{n}}\right] \mathbf{v}
$$

where all the matrices are symmetric,

$$
A^{0}(\varepsilon \mathbf{v}):=\left(\begin{array}{cc}
\frac{\mathcal{P}^{\prime}\left(\rho_{0}+\varepsilon r\right)}{\rho_{0}+\varepsilon r} & \mathbf{0} \\
\mathbf{0} & \left(\rho_{0}+\varepsilon r\right) I
\end{array}\right)
$$

is also positive definite, and the matrices $C_{j}$ multiplied by $\frac{1}{\varepsilon}$ and $K_{m, n}$ in the second-derivative terms are constant. Uniform estimates for solutions of (5.4) can therefore be obtained by taking some derivative $\partial_{\mathbf{x}}^{\alpha}:=\partial_{x_{1}}^{\alpha_{1}} \cdots \partial_{x_{d}}^{\alpha_{d}}$ of the equation (5.4), multiplying by $\partial_{\mathbf{x}}^{\alpha} \mathbf{v}$, and integrating over space, since the large terms $\frac{1}{\varepsilon} \int \mathbf{v} \cdot C_{j} \mathbf{v}_{x_{j}}$ equal $\frac{1}{2 \varepsilon} \int \partial_{x_{j}}\left(\mathbf{v} \cdot C_{j} \mathbf{v}\right)$ and so vanish identically, while terms involving the $O\left(\frac{1}{\varepsilon}\right)$ expression $\mathbf{v}_{t}$ are always multiplied by a factor of $\varepsilon$ on account of the $\varepsilon$ in $A_{0}(\varepsilon \mathbf{v})$, and so are actually of order one. Adding the resulting estimates for $\frac{d}{d t}\left(\partial_{\mathbf{x}}^{\alpha} \mathbf{v}, A_{0}(\varepsilon \mathbf{v}) \partial_{\mathbf{x}}^{\alpha} \mathbf{v}\right)$ over $0 \leq|\alpha|:=\sum_{j}\left|\alpha_{j}\right| \leq s$ with $s$ sufficiently large yields a closed uniform set of estimates for a quantity equivalent to the $H^{s}$ norm. 
In the "slow" case in which the initial data are "well-prepared", i.e., satisfy $\nabla_{\mathbf{x}} r_{0, \varepsilon}=O(\varepsilon)=\nabla_{\mathbf{x}} \cdot \mathbf{u}_{0, \varepsilon}$, or more generally $\sum_{j} C_{j} \partial_{x_{j}} \mathbf{v}_{0, \varepsilon}=O(\varepsilon)$, so that $\mathbf{v}_{t}$ is uniformly bounded at time zero, uniform bounds are also obtained for the time derivative of

$$
\left(\partial_{\mathbf{x}}^{\alpha} \mathbf{v}_{t}, A_{0} \partial_{\mathbf{x}}^{\alpha} \mathbf{v}_{t}\right)
$$

for $|\alpha| \leq s-1$, thereby completing the required set of estimates. In this case the limit profile $\mathbf{v}^{0}$ is purely slow, so that (5.3) is a convergence result in the ordinary sense.

However, when the initial data are not well prepared, then (5.5) is not uniformly bounded initially, so no uniform estimate is feasible. Fortunately, applying $\mathbb{S}\left(-\frac{t}{\varepsilon}\right)$ to $(5.4)$, where $\mathbb{S}$ is the solution operator to the "fast" equation

$$
A_{0}(\mathbf{0}) \mathbf{v}_{\tau}+\sum_{j} C_{j} \mathbf{v}_{x_{j}}=0
$$

cancels the the large term $\sum_{j} C_{j} \mathbf{v}_{x_{j}}$ so that uniform $H^{s-1}$ estimates for $\partial_{t} \mathbb{S}\left(-\frac{t}{\varepsilon}\right) \mathbf{v}$ are obtained directly from the resulting PDE. More conveniently, applying $\mathbb{S}\left(\tau-\frac{t}{\varepsilon}\right)$ instead yields uniform estimates for $\partial_{t} \mathbb{S}\left(\tau-\frac{t}{\varepsilon}\right) \mathbf{v}$, thereby introducing the variable $\tau$ in such a way that the original solution is restored for $\tau=\frac{t}{\varepsilon}$, as desired. Note that for the rescaled barotropic Navier-Stokes equations (5.1)-(5.2), $A_{0}(\mathbf{0})=\mathcal{P}^{\prime}\left(\rho_{0}\right) \tilde{A}$ and $\sum_{j} C_{j} \partial_{x_{j}}=-\mathcal{P}^{\prime}\left(\rho_{0}\right) \mathbb{L}$, so that (5.6) is equivalent to (4.7) and hence $\mathbb{S}$ is the same as defined earlier. By the unitarity of $\mathbb{S}$ with respect to the inner product defined by $A_{0}(\mathbf{0})$, the uniform estimates obtained for $\mathbf{v}$ imply the same bounds for $\mathbb{S}\left(\tau-\frac{t}{\varepsilon}\right) \mathbf{v}$, which together with the estimates obtained for the time derivative of that expression yield the compactness necessary to obtain convergence.

\section{EXTENSIONS}

\subsection{Improved error estimate and asymptotic expansions}

When can the $o(1)$ error estimate in Theorem 5.1 or its variants be improved to $\mathbf{v}^{\varepsilon}-\mathbf{v}^{0}+O(\varepsilon)$ or even to an asymptotic expansion $\mathbf{v}^{\varepsilon}=\mathbf{v}^{0}+\varepsilon \mathbf{v}^{1}+\cdots+\varepsilon^{k} \mathbf{v}^{k}+O\left(\varepsilon^{k+1}\right)$ ? Such results cannot be expected to hold true in general because of small divisor problems in the periodic case or slow decay of the fast part or the pressure in the whole-space case. Nevertheless, such results have been proven when in the slow case $[29,43]$, i.e., when $\mathbf{v}_{\text {fast }}^{0}=0$, and for generic values of the ratios of the spatial periods in the periodic case $[16,25,44]$.

\subsection{Boundaries: Euler equations}

For the slow case, results analogous to Theorem 5.1 have been proven for inviscid flow in bounded domains in $[3,15,42]$. More recently, the fast case was treated in [45].

For moving domains, the formal asymptotics of solutions have been calculated [2], while for domains with open boundaries uniform estimates have been derived for the linearized system [17].

\subsection{Fast decay of fast waves in the whole space}

In dimensions larger than one, solutions of the wave equation (4.25) having initial data in $H^{s} \cap L^{1}$ decay in $L^{\infty}$. As a consequence, the fast part of the solution decays on acoustic time scale, and so is negligible on the convective time scale. Thus, $\mathbf{v}^{\varepsilon}$ converges on compact sets to a slow limit, albeit nonuniformly in time for $t$ near zero. This result was proven for solutions in $\mathbb{R}^{d}$ having a single spatial scale in $[23,49]$, and has been extended to exterior domains [22], including the case when there is a nonvanishing steady flow at infinity [24], and also to a half-space $\mathbb{R}_{+}^{d}[21]$.

\subsection{Viscous flows: weak solutions and global solutions}

In the energy estimates derived above for (5.4), we only made use of the fact that the viscous terms do not hinder the essentially hyperbolic estimates that are valid when viscosity is absent. Of course, those viscous terms actually contribute very helpful terms to those estimates. As for the incompressible equations, estimates 
more complicated than those described above make it possible to take advantage of those helpful terms so as to allow the initial data to be less smooth, and to obtain global existence of weak solutions for small $\varepsilon$ when the limit solution exists for all time $[5,10,11,18-20,33,35,36]$.

Surprisingly, when both fast waves and boundaries are present then the interaction of fast waves with a boundary layer usually makes them decay fast in bounded domains, so that $v^{\varepsilon}$ converges to the solution of the slow equations [12].

Even in the inviscid case the limit solution sometimes exists for all time. Without viscosity one cannot expect the full solution $\mathbf{v}^{\varepsilon}$ to also exist for all time, but its time of existence has been shown in various cases to tend to infinity as $\varepsilon \rightarrow 0[13,46]$.

\subsection{Entropy}

For non-isentropic inviscid flow with one spatial scale the rescaled equations (3.15)-(3.17) reduce to

$$
\begin{array}{r}
a_{1}^{0}\left(\mathcal{P}_{0}+\varepsilon p, s\right)\left[p_{t}+\mathbf{u} \cdot \nabla p\right]+\frac{1}{\varepsilon} \nabla \cdot \mathbf{u}=0 \\
a_{2}^{0}\left(\mathcal{P}_{0}+\varepsilon p, s\right)\left[\mathbf{u}_{t}+(\mathbf{u} \cdot \nabla) \mathbf{u}\right]+\frac{1}{\varepsilon} \nabla p=0 \\
s_{t}+\mathbf{u} \cdot \nabla s=0
\end{array}
$$

where

$$
a_{1}^{0}:=\frac{1}{\rho} \frac{\partial \rho}{\partial \mathcal{P}}, \quad a_{2}^{0}:=\rho .
$$

These equations almost have the form (5.4) with the additional spatial variable and viscous terms omitted, except that $A^{0}:=\left(\begin{array}{cc}a_{1}^{0} & 0 \\ 0 & a_{2}^{0} I\end{array}\right)$ now depends on $\mathbf{v}$ as well as on $\varepsilon \mathbf{v}$, i.e., (5.4) is replaced by

$$
A_{0}(\varepsilon \mathbf{v}, \mathbf{v}) \mathbf{v}_{t}+\sum_{j} A_{j}(\mathbf{v}, \varepsilon) \mathbf{v}_{x_{j}}+\frac{1}{\varepsilon} \sum_{j} C_{j} \mathbf{v}_{x_{j}}=0
$$

In general, solutions of (6.2) having uniformly bounded initial data do not exist for a time independent of $\varepsilon$, as can be seen from the explicitly-solvable equation $\left(1+v^{2}\right) v_{t}+\frac{1}{\varepsilon} v_{x}=0$. In terms of energy estimates, the difficulty with (6.2) is that the equation for $\mathbf{v}_{x_{j}}$ has the form

$$
A^{0}(\varepsilon \mathbf{v}, \mathbf{v}) \mathbf{v}_{x_{j} t}+\left[\left(\mathbf{v}_{x_{j}} \cdot \nabla_{\mathbf{v}}\right) A^{0}(\varepsilon \mathbf{v}, \mathbf{v})\right] \mathbf{v}_{t}+\varepsilon\left[\left(\mathbf{v}_{x_{j}} \cdot \nabla_{\varepsilon \mathbf{v}}\right) A^{0}(\varepsilon \mathbf{v}, \mathbf{v})\right] \mathbf{v}_{t}+\cdots=0,
$$

in which the $O\left(\frac{1}{\varepsilon}\right)$ term $\mathbf{v}_{t}$ is not always multiplied by a compensating factor of $\varepsilon$, as holds for (5.4). Of course, in the slow case for which $\mathbf{v}_{t}$ is bounded initially this problem does not arise, so that uniform estimates can be obtained $[42,43]$.

The Euler equations have a special structure beyond that of (6.2). Among other features, $A^{0}$ depends on $\mathbf{v}$ rather than $\varepsilon \mathbf{v}$ only through its dependence on $s$, whose time-derivative equation contains no large $O\left(\frac{1}{\varepsilon}\right)$ terms. In other words, although the fast operator is nonlinear it depends only on a slow variable. Taking into account certain other special features of the equations as well then permits a complicated set of estimates to be obtained, which yield uniform bounds for

$$
p, \mathbf{u}, s, s_{t} \text {, and } \mathbb{P}\left[a_{2}^{0} \mathbf{u}\right]_{t} .
$$

In particular, even in the fast case solutions of the Euler equations with uniformly bounded initial data exist for a time independent of $\varepsilon$. Nevertheless, the equations exhibit non-uniform stability in that small changes in $s$ may cause an $O(1)$ change in $\mathbf{u}$. Furthermore, on account of the nonlinearity of the fast operator, explicit Fourier-space computations like those of Section 4 are not possible. 
The uniform bounds for the quantities in (6.3) imply that, after restricting to a sequence of values of $\varepsilon, \mathbf{u}^{\varepsilon}$ converges weakly to some $\mathbf{u}_{\text {slow }}^{0}, p^{\varepsilon}$ converges weakly to some $p_{\text {slow }}^{0}, s^{\varepsilon}$ converges strongly to some $s^{0}$, and $\mathbb{P}\left[a_{2}^{0}\left(\mathcal{P}_{0}+\varepsilon p^{\varepsilon}, s^{\varepsilon}\right) \mathbf{u}^{\varepsilon}\right]$ converges strongly to $\mathbb{P}\left[a_{2}^{0}\left(\mathcal{P}_{0}, s^{0}\right) \mathbf{u}_{\text {slow }}^{0}\right]$.

In all $\mathbb{R}^{d}$, if the initial data decay sufficiently rapidly at infinity then the fast waves still decay quickly, so that the limit satisfies the "stratified" incompressible Euler equations in which the entropy, and hence also the density, remain non-constant [38]. An extension of this result to exterior domains has recently been obtained $[1]$.

In the periodic case, the above convergence results suffice to obtain the equations

$$
\begin{gathered}
\nabla p_{\text {slow }}^{0}=0=\nabla \cdot \mathbf{u}_{\text {slow }}^{0} \\
s_{t}^{0}+\mathbf{u}_{\text {slow }}^{0} \cdot \nabla s^{0}=0 \\
\partial_{t} p_{\text {slow }}^{0}=0
\end{gathered}
$$

for the limit variables [39]. However, in order to obtain a closed set of equations we still need to find the equation satisfied by $\mathbf{u}_{\text {slow }}^{0}$, which turns out not to be just the incompressible Euler equation in general. Rather, a formal calculation of the equation for $\mathbf{u}_{\text {slow }}^{0}$ shows that that equation includes an extra term involving the limit of quadratic expressions in the fast part of the solution, which tends weakly to zero.

In the very special case of only one spatial dimension, the limit can be both calculated completely and justified [39]. The limit equation for $u_{\text {slow }}^{0}$ turns out to be simply

$$
\partial_{t} u_{\text {slow }}^{0}=0
$$

However, this result depends on the fact that

$$
a_{1}^{0}=\frac{1}{a_{2}^{0}} \frac{\partial a_{2}^{0}}{\partial \mathcal{P}}
$$

as follows from (6.1). If we consider general positive functions $a_{j}^{0}$ depending on $\left(\mathcal{P}_{0}+\varepsilon p, s\right)$ then complicated equations link $u_{\text {slow }}^{0}$ to the weak limits of $\mathrm{e}^{ \pm i \alpha_{j} t / \varepsilon}\left(\begin{array}{l}p^{\varepsilon} \\ u^{\varepsilon}\end{array}\right)$, where the $\alpha_{j}^{2}$ are the nonzero eigenvalues of the operator

$$
-\frac{1}{a_{1}^{0}\left(\mathcal{P}_{0}, s(0, x)\right)} \partial_{x}\left[\frac{1}{a_{2}^{0}\left(\mathcal{P}_{0}, s(0, x)\right)} \partial_{x}\right] \text {. }
$$

The key point allowing the formula so obtained to be justified is that, after an appropriate transformation, the spectral decomposition of the corresponding operator for non-zero $\varepsilon$ is independent of $\varepsilon$ and $t$.

In the multi-dimensional case the formal calculation of the extra term in the limit, which once again involves the spectral decomposition of the fast operator, assumes that the spectrum of that fast operator is simple and non-resonant. For certain finite-dimensional truncations of the equations those assumptions can be shown to be generic and to ensure convergence to the limit equations.

\section{SOME OPEN PROBLEMS}

\subsection{Uniformly locally bounded initial data}

The uniformly local Sobolev space $H_{u l}^{s}$ is the space of functions having a finite value of the norm

$$
\|f\|_{H_{u l}^{s}}:=\sqrt{\sup _{\mathbf{x}_{0}} \sum_{0 \leq|\alpha| \leq s} \int_{\mathbb{R}^{d}} \psi\left(\mathbf{x}-\mathbf{x}_{0}\right)\left[D^{\alpha} f(x)\right]^{2} \mathrm{~d} x}
$$


where it is convenient to choose $\psi(\mathbf{x}):=\mathrm{e}^{-\sqrt{1+|\mathbf{x}|^{2}}}$ instead of being a $C_{0}^{\infty}$ function as is customary. Symmetric hyperbolic equations are well-posed in these spaces [27]. Suppose that the initial data for (5.1)-(5.2) only belong to $H_{u l}^{s}$ rather than $H^{s}$. Are there cases for which the corresponding solutions exist for a time independent of $\varepsilon$ and satisfy uniform estimates? The difficulty in extending previous results to this case is that the large $O\left(\frac{1}{\varepsilon}\right)$ terms are no longer anti-symmetric with respect to weighted $L^{2}$ norms, and so do not automatically drop out of the energy estimates.

In the one-dimensional case, some analogous results for more general systems have been proven for the BV norm in [8]. Furthermore, uniform bounds in $H^{s}$ norms can be obtained by using the explicit solution of the fast operator: For simplicity, consider the slightly simplified equations

$$
r_{t}+u r_{x}+\frac{1}{\varepsilon} u_{x}=0, \quad u_{t}+u u_{x}+\frac{1}{\varepsilon} r_{x}=0 .
$$

In terms of the transformed variables

$$
v^{ \pm}(t, x):=r\left(t, x \pm \frac{t}{\varepsilon}\right) \pm u\left(t, x \pm \frac{t}{\varepsilon}\right)
$$

occurring in the formula for the fast solution operator, (7.1) becomes

$$
v_{t}^{ \pm}+u\left(t, x \pm \frac{t}{\varepsilon}\right) v_{x}^{ \pm}=0 .
$$

Since $u$ can be recovered from $v^{ \pm}$via

$$
u(t, x)=\frac{1}{2}\left[v^{+}\left(t, x-\frac{t}{\varepsilon}\right)-v^{-}\left(t, x+\frac{t}{\varepsilon}\right)\right],
$$

(7.2) yields uniform uniformly local estimates for $v^{ \pm}$, and hence also for $r$ and $u$, since $r$ can likewise be recovered from $v^{ \pm}$.

The key to these one-dimensional estimates was that the fast solution operator is uniformly bounded in $L^{\infty}$. This no longer holds true in higher dimensions, which implies that uniform estimates cannot hold in the fast case. For example, in three dimensions the solution at any point to the wave equation with initial value zero and initial derivative $g$ is a constant times $\frac{1}{t}$ times the integral of $g$ over the sphere of radius $t$ centered at that point. Hence if $g$ were identically one then the solution to the wave equation would grow linearly in the fast time. However, just as in one dimension, $g$ cannot be identically one for solutions $r$ for which the wave equation is derived from the fast equations

$$
r_{\tau}+\nabla \cdot \mathbf{u}=0, \quad \mathbf{u}_{\tau}+\nabla r=0
$$

because the initial value of $r_{\tau}$ is a sum of spatial derivatives. Nevertheless, it is possible for uniformly local initial data $\mathbf{u}(0, \mathbf{x})$ to have $g:=\nabla \cdot \mathbf{u}(0, \mathbf{x})$ equal one on a series of concentric circles whose radii tend to infinity, which still yields non-uniformly bounded solutions.

Although this shows that uniform bounds cannot hold in higher dimensions in the fast case, it does not immediately preclude such estimates in the slow case, provided that fast waves are absent initially through some order in $\varepsilon$ that depends on the dimension. Do uniform bounds hold in higher dimensions for sufficiently well-prepared initial data?

\subsection{Multiple spatial scales for fast non-isentropic flow}

The estimates of [38] for fast non-isentropic flow do not work with multiple spatial scales, because the condition (1.3) there no longer holds. However, multiple spatial scales are especially interesting for such flows because the formal asymptotics calculated in [31] shows that, unlike the barotropic case discussed here, the small-scale flow does affect the large-scale one. Do fast non-isentropic solutions with multiple spatial scales exist for a time independent of $\varepsilon$ and satisfy uniform bounds? 


\subsection{Genericity of simple non-resonant spectrum for fast periodic non-isentropic flow}

Do the genericity and convergence results proven in [39] for finite-dimensional truncations of the nonisentropic Euler equations also hold for the full Euler equations?

Acknowledgements. I thank Guy Métivier for the pleasure of working with him on [38, 39] and for encouragement in writing this review. Thanks also to Thomas Alazard and to Rupert Klein for interesting conversations.

\section{REFERENCES}

[1] T. Alazard, Incompressible limit of the nonisentropic Euler equations with the solid wall boundary conditions. Adv. Differential Equations, to appear.

[2] G. Alì, Low Mach number flows in time-dependent domains. SIAM J. Appl. Math. 63 (2003) 2020-2041.

[3] K. Asano, On the incompressible limit of the compressible euler equation. Japan J. Appl. Math. 4 (1987) 455-488.

[4] B.J. Bayly, C.D. Levermore and T. Passot, Density variations in weakly compressible flows. Phys. Fluids A 4 (1992) $945-954$.

[5] D. Bresch, B. Desjardins, E. Grenier and C.-K. Lin, Low Mach number limit of viscous polytropic flows: formal asymptotics in the periodic case. Stud. Appl. Math. 109 (2002) 125-149.

[6] G. Browning and H.-O. Kreiss, Problems with different time scales for nonlinear partial differential equations. SIAM J. Appl. Math. 42 (1982) 704-718.

[7] G. Browning, A. Kasahara and H.-O. Kreiss, Initialization of the primitive equations by the bounded derivative method. J. Atmospheric Sci. 37 (1980) 1424-1436.

[8] C. Cheverry, Justification de l'optique géométrique non linéaire pour un système de lois de conservation. Duke Math. J. 87 (1997) 213-263.

[9] A. Chorin, A numerical method for solving incompressible viscous flow problems. J. Comput. Phys. 2 (1967) $12-26$.

[10] R. Danchin, Zero Mach number limit for compressible flows with periodic boundary conditions. Amer. J. Math. 124 (2002) $1153-1219$

[11] B. Desjardins and E. Grenier, Low Mach number limit of viscous compressible flows in the whole space. R. Soc. Lond. Proc. Ser. A Math. Phys. Eng. Sci. 455 (1999) 2271-2279.

[12] B. Desjardins, E. Grenier, P.-L. Lions and N. Masmoudi, Incompressible limit for solutions of the isentropic navier-stokes equations with dirichlet boundary conditions. J. Math. Pures Appl. 78 (1999) 461-471.

[13] A. Dutrifoy and T. Hmidi, The incompressible limit of solutions of the two-dimensional compressible Euler system with degenerating initial data. C. R. Math. Acad. Sci. Paris 336 (2003) 471-474.

[14] D. Ebin, The motion of slightly compressible fluids viewed as a motion with strong constraining force. Ann. Math. 105 (1977) $141-200$.

[15] D. Ebin, Motion of slightly compressible fluids in a bounded domain I. Comm. Pure Appl. Math. 35 (1982) $451-485$.

[16] I. Gallagher, Asymptotic of the solutions of hyperbolic equations with a skew-symmetric perturbation. J. Differential Equations 150 (1998) 363-384.

[17] B. Gustafsson and H. Stoor, Navier-Stokes equations for almost incompressible flow. SIAM J. Numer. Anal. 28 (1991) 1523-1547.

[18] T. Hagstrom and J. Lorenz, All-time existence of classical solutions for slightly compressible flows. SIAM J. Math. Anal. 29 (1998) 652-672.

[19] T. Hagstrom and J. Lorenz, On the stability of approximate solutions of hyperbolic-parabolic systems and the all-time existence of smooth, slightly compressible flows. Indiana Univ. Math. J. 51 (2002) 1339-1387.

[20] D. Hoff, The zero-Mach limit of compressible flows. Comm. Math. Phys. 192 (1998) 543-554.

[21] T. Iguchi, The incompressible limit and the initial layer of the compressible Euler equation in $R_{+}^{n}$. Math. Methods Appl. Sci. 20 (1997) 945-958.

[22] H. Isozaki, Singular limits for the compressible Euler equation in an exterior domain. J. Reine Angew. Math. 381 (1987) 1-36.

[23] H. Isozaki, Wave operators and the incompressible limit of the compressible Euler equation. Comm. Math. Phys. 110 (1987) 519-524.

[24] H. Isozaki, Singular limits for the compressible Euler equation in an exterior domain. II. Bodies in a uniform flow. Osaka J. Math. 26 (1989) 399-410.

[25] J.-L. Joly, G. Métivier and J. Rauch, Coherent and focusing multidimensional nonlinear geometric optics. Ann. Sci. École Norm. Sup. (4) 28 (1995) 51-113.

[26] J.-L. Joly, G. Métivier and J. Rauch, Dense oscillations for the compressible 2-d Euler equations, in Nonlinear partial differential equations and their applications. Collège de France Seminar, Vol. XIII (Paris, 1994/1996), Longman, Harlow. Pitman Res. Notes Math. Ser. 391 (1998) 134-166.

[27] T. Kato, The Cauchy problem for quasi-linear symmetric hyperbolic systems. Arch. Rational Mech. Anal. 58 (1975) 181-205. 
[28] S. Klainerman and A. Majda, Singular perturbations of quasilinear hyperbolic systems with large parameters and the incompressible limit of compressible fluids. Comm. Pure Appl. Math. 34 (1981) 481-524.

[29] S. Klainerman and A. Majda, Compressible and incompressible fluids. Comm. Pure Appl. Math. 35 (1982) 629-653.

[30] R. Klein, Semi-implicit extension of a Godunov-type scheme based on low Mach number asymptotics. I. One-dimensional flow. J. Comput. Phys. 121 (1995) 213-237.

[31] R. Klein, N. Botta, T. Schneider, C.-D. Munz, S. Roller, A. Meister, L. Hoffmann and T. Sonar, Asymptotic adaptive methods for multi-scale problems in fluid mechanics. J. Engrg. Math. 39 (2001) 261-343.

[32] H.-O. Kreiss, Problems with different time scales for partial differential equations. Comm. Pure Appl. Math. 33 (1980) $399-439$.

[33] C.K. Lin, On the incompressible limit of the compressible navier-stokes equations. Comm. Partial Differential Equations 20 (1995) 677-707.

[34] P.-L. Lions, Mathematical topics in fluid mechanics, Vol. 1, Oxford Lecture Series in Mathematics and its Applications. The Clarendon Press Oxford University Press, New York 3 (1996).

[35] P.-L. Lions and N. Masmoudi, Incompressible limit for a viscous compressible fluid. J. Math. Pures Appl. 77 (1998) 585-627.

[36] P.-L. Lions and N. Masmoudi, Une approche locale de la limite incompressible. C. R. Acad. Sci. Paris Sér. I Math. 329 (1999) 387-392.

[37] A. Meister, Asymptotic single and multiple scale expansions in the low Mach number limit. SIAM J. Appl. Math. 60 (2000) $256-271$.

[38] G. Métivier and S. Schochet, The incompressible limit of the non-isentropic euler equations. Arch. Rational Mech. Anal. 158 (2001) 61-90.

[39] G. Métivier and S. Schochet, Averaging theorems for conservative systems and the weakly compressible Euler equations. J. Differential Equations 187 (2003) 106-183.

[40] B. Müller, Low-Mach-number asymptotics of the Navier-Stokes equations. J. Engrg. Math. 34 (1998) 97-109.

[41] M. Schiffer, Analytical theory of subsonic and supersonic flows, in Handbuch der Physik. Springer-Verlag, Berlin 9 (1960) $1-161$.

[42] S. Schochet, The compressible Euler equations in a bounded domain: existence of solutions and the incompressible limit. Comm. Math. Phys. 104 (1986) 49-75.

[43] S. Schochet, Asymptotics for symmetric hyperbolic systems with a large parameter. J. Differential Equations 75 (1988) 1-27.

[44] S. Schochet, Fast singular limits of hyperbolic PDEs. J. Differential Equations 114 (1994) 476-512.

[45] P. Secchi, On the singular incompressible limit of inviscid compressible fluids. J. Math. Fluid Mech. 2 (2000) $107-125$.

[46] T. Sideris, The lifespan of smooth solutions to the three-dimensional compressible Euler equations and the incompressible limit. Indiana Univ. Math J. 40 (1991) 535-550.

[47] L. Sirovich, Initial and boundary value problems in dissipative gas dynamics. Phys. Fluids 10 (1967) 24-34.

[48] R. Temam, Navier-Stokes equations. Theory and numerical analysis. North-Holland Publishing Co., Amsterdam (1977).

[49] S. Ukai, The incompressible limit and initial layer of the compressible Euler equation. J. Math. Kyoto U. 26 (1986) 323-331.

[50] P.S. van der Gulik, The linear pressure dependence of the viscosity at high densities. Physica A 256 (1998) 39-56.

[51] M. Van Dyke, Perturbation methods in fluid mechanics. Appl. Math. Mech. 8. Academic Press, New York (1964).

[52] G.P. Zank and W.H. Matthaeus, The equations of nearly incompressible fluids. I. Hydrodynamics, turbulence, and waves. Phys. Fluids A 3 (1991) 69-82. 